Bundesgesundheitsbl -

Leitthema:Mutter-Kind-Übertragung von Infektionskrankheiten

U. Marcus · Robert Koch-Institut, Berlin

\title{
AIDS und HIV-Infektionen bei Frauen und Kindern in Deutschland
}

\section{Zusammenfassung}

Der Anteil von Frauen an den HIV-Infizierten und AIDS-Patienten in Deutschland ist - wie in anderen Industriestaaten - deutlich geringer als der der Männer. Die unterschiedliche Betroffenheit von Frauen in den Industrie- und Entwicklungsländern ist auf soziale, kulturelle und ökonomische Ursachen zurückzuführen. Im Verlauf der HIV-Epidemie in Deutschland hat der Anteil von Frauen an den Infizierten langsam, aber stetig zugenommen und liegt derzeit bei etwa $20 \%$. Auch die Infektionsrisiken haben sich gewandelt: Anfangs war die Mehrheit der weiblichen Infizierten i.v. drogenabhängig, seit Anfang der neunziger Jahre ist der heterosexuelle Übertragungsweg der bedeutendste Infektionsweg. Trotz der zahlenmäßigen Zunahme HIV-infizierter Frauen sank die Zahl perinatal mit HIV infizierter Kinder in den vergangenen fünf Jahren. Dies ist auf eine Kombination medikamentöser Prophylaxe und geburtshilflicher Maßnahmen zurückzuführen, die sich in Deutschland seit 1994 als Standardvorgehen etabliert haben. Mit diesem Vorgehen konnte das Übertragungsrisiko von Mutter zu Kind auf unter 2\% gesenkt werden. Da die Maßnahmen nur bei Kenntnis der HIV-Infektion der Mutter eingesetzt werden können, sollte möglichst allen Schwangeren ein HIV-Antikörpertest mit entsprechender Aufklärung und Beratung angeboten werden.
D ie HIV-Epidemie in Deutschland ist nach wie vor eine von Männern dominierte Epidemie, obwohl sich der Anteil der Frauen an den HIV-Infizierten und an AIDS Erkrankten im Laufe der Jahre kontinuierlich erhöht hat. Die männliche Dominanz ist Folge der weitgehenden Beschränkung der HIV-Epidemie auf bestimmte Betroffenengruppen, die, wie homosexuelle Männer und Hämophile, quasi definitionsgemäß nur Männer umfassen, oder, wie intravenöse Drogengebraucher, in ihrer Zusammensetzung mehrheitlich von Männern gestellt werden.

Weibliche Homosexuelle tragen ein offenbar vernachlässigbar geringes Risiko, sich bei gleichgeschlechtlichen Sexualkontakten mit HIV zu infizieren vermutlich, weil in der Regel nur geringe Mengen an potentiell infektiösen Körperflüssigkeiten ausgetauscht werden und/oder unspezifische Protektivfaktoren wie z.B. der saure $\mathrm{pH}$-Wert des Scheidensekretes stärker zum Tragen kommen.

\section{Unterschiedliche Betroffenheit von Frauen in Industrie- und Entwicklungsländern}

Weibliche Prostituierte, die in vielen Ländern die am stärksten durch HIV gefährdete Gruppe darstellen (HIV-Prävalenzen von 50 bis $80 \%$ sind in vielen $\mathrm{Re}$ gionen bzw. Städten Schwarzafrikas und
Südostasiens keine Seltenheit), sind in Deutschland bislang kaum von der HIVEpidemie betroffen, es sei denn, es handelt sich um drogengebrauchende Beschaffungsprostituierte. Ursache hierfür dürfte die größere Professionalisierung des Prostitutionssektors in den westlichen Industriestaaten sein, die mit einem größeren Selbstbewußtsein und einer stärkeren Fähigkeit zur Durchsetzung eigener Schutzinteressen gegenüber den Freiern einhergeht und es den Frauen eher ermöglicht, auf der Verwendung von Kondomen zu bestehen.

\section{„Obwohl HIV spätestens seit Mitte der achtziger Jahre im Bereich der Beschaffungsprostitution präsent ist, war dies bislang offenbar nicht ausreichend, um eine eigenständige heterosexuelle Epidemie zu starten."}

Außerdem gibt es offenbar weitere soziale und kulturelle Unterschiede zwischen den Industriestaaten und Entwicklungsländern, die sich auf die durchschnittlichen Frequenzen von Prostitutionskontakten auswirken und damit die Ausbreitungsbedingungen für Erreger wie HIV beeinflussen. Diese Un-

\section{Dr. Ulrich Marcus}

Robert Koch-Institut, Postfach 650280, D-13302 Berlin 
Bundesgesundheitsbl -

Gesundheitsforsch - Gesundheitsschutz

1999 - 42: 553-557 @ Springer-Verlag 1999

\section{U.Marcus}

\section{AIDS and HIV infections in women and children in Germany}

\section{Summary}

The proportion of women among people with HIV infection and AIDS in Germany is considerably smaller than the proportion of men. The differential extent of the HIV epidemic in women in developed and developing countries is caused by certain social, cultural and economic conditions. During the course of the HIV epidemic in Germany the proportion of females among the infected persons has increased slowly, but steadily and has reached $20 \%$ by now. Also the transmission risks have changed. In the beginning of the epidemic the majority of women with HIV infection were IDU (intravenous drug users), since the nineties the majority has been infected by heterosexual intercourse. Despite the increase of HIV infected females the number of perinatally infected children has been decreasing in the last five years. This is because a combination of antiretroviral prophylactic therapy with primary cesarean section as the mode of delivery has been established as the standard procedure in Germany. With this procedure the transmission risk from mother to children declined to below $2 \%$. Because these measures can only be used, if the HIV status of the pregnant women is known, HIV antibody testing with adequate counselling should be offered to all pregnant women.

\section{Leitthema: Mutter-Kind-Übertragung von Infektionskrankheiten}

terschiede hängen u.a. mit der starken Binnenmigration in Entwicklungsländern zusammen, die zur Trennung der Arbeiter von ihren Familien führt, aber auch - paradoxerweise - mit einer konservativen Sexualmoral, die es „anständigen“ jungen Frauen nicht gestattet, vorehelichen Geschlechtsverkehr $\mathrm{zu}$ praktizieren, während es jungen Männern in der Regel erlaubt ist, ihre ersten sexuellen Erfahrungen mit Prostituierten zu machen.

Eine wichtige Rolle spielt die generell größere ökonomische Abhängigkeit der Frauen von Männern auch außerhalb des unmittelbaren Prostitutionskontextes. Für junge Frauen stellen sexuelle Beziehungen zu älteren, ökonomische Sicherheit bietenden Männern oft eine überlebensnotwendige Form der Existenzsicherung dar. Sexuelle Beziehungen mit großen Altersunterschieden erlauben eine raschere Ausbreitung sexuell übertragbarer Erreger, während geringere Altersdifferenzen in den westlichen Industriestaaten zu Alterskohorteneffekten führen, bei denen die jeweils nachwachsenden Altersgruppen zunächst relativ niedrige Infektionsprävalenzen aufweisen.

Eine nicht unwichtige Rolle dürften darüber hinaus die geringere Belastung der im Prostitutionsbereich arbeitenden Frauen mit anderen sexuell übertragbaren Infektionen spielen, die hierzulande schneller erkannt und wirksamer therapiert werden.

\section{Veränderung der Infektions- risiken von Frauen im Verlauf der HIV-Epidemie}

Die Infektionsrisiken von Frauen haben sich im Laufe der Epidemie deutlich gewandelt: bis zum Beginn der neunziger Jahre stellten aktuell oder ehemals drogengebrauchende Frauen die Mehrheit der weiblichen AIDS-Patienten dar. Bis einschließlich 1990 lag der Anteil von Frauen an allen AIDS-Patienten noch unter $10 \%$. Bei den insgesamt noch relativ niedrigen Fallzahlen spielten transfusionsbedingte HIV-Infektionen und AIDS-Erkrankungen in diesen Jahren auch noch eine anteilsmäßig wichtige Rolle [1] (Abb. 1).
Bei den Infektionsrisiken der männlichen Partner, von denen die Frauen mit heterosexuellen Kontakten ihre HIV-Infektion erworben haben, geht erwartungsgemäß ebenfalls das Risiko über Blut und Blutprodukte zurück. Keine eindeutigen Auf- oder Abwärtstrends zeigen sich bislang bezüglich des Anteils der Frauen, die sich über bisexuelle Partner, drogengebrauchende Partner oder Partner aus Entwicklungsländern infizieren bzw. deren einheimische Partner sich ihrerseits auf heterosexuellem Wege infiziert haben (10 bis $20 \%$ drogengebrauchende Partner, 5 bis 10\% Partner aus Entwicklungsländern, ca. 5\% Partner mit heterosexuellem Risiko).

\section{„Bei Frauen hat das Infektionsrisiko über heterosexuelle Kontakte zugenommen."}

Kontinuierlich hat allerdings in den vergangenen Jahren der Anteil an Frauen zugenommen, die über das mögliche Infektionsrisiko ihres als Infektionsquelle in Frage kommenden Partners keine Aussagen machen können oder wollen (Abb. 2).

Bei den HIV-Neudiagnosen stellen Frauen inzwischen 20\% der Meldungen. Dieser Anteil wurde Anfang der neunziger Jahre erreicht und hat sich seitdem nicht mehr wesentlich gesteigert. An Infektionsrisiken liegen bei den Neudiagnosen heterosexuelle Kontakte und Herkunft aus Hochprävalenzregionen in Entwicklungsländern gleichermaßen vor, die Gruppe der über i.v.-Drogengebrauch infizierten Frauen ist mittlerweile auf den dritten Platz zurückgefallen (Abb. 3, 4).

\section{HIV-Infektionen bei Frauen und Mutter-Kind-Übertragung}

Das Durchschnittsalter der infizierten und erkrankten Frauen liegt deutlich niedriger als bei Männern, was mit dem im Vergleich zu heterosexuellen STDEpidemien höheren Durchschnittsalter der homosexuellen HIV-Infizierten und dem relativ niedrigen Alter der infizierten i.v.-Drogenabhängigen in Europa erklärbar ist. 


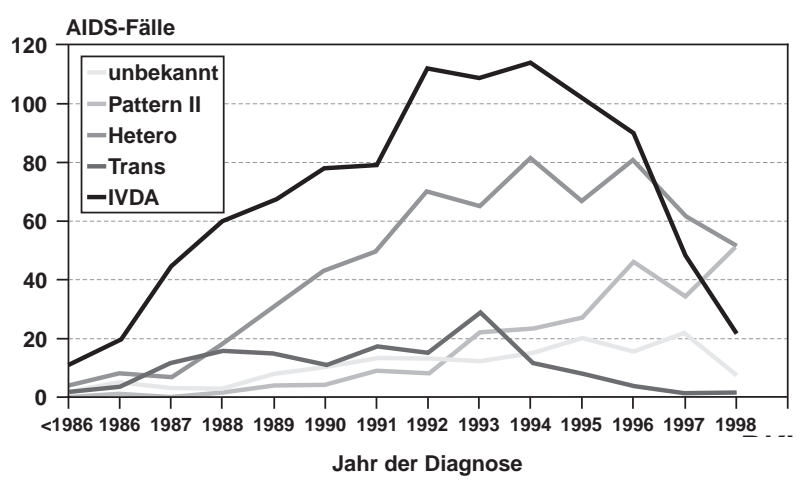

Abb. 1 $\triangle$ AIDS-Fälle bei Frauen in Deutschland nach Infektionsrisiko und Jahr der Diagnose

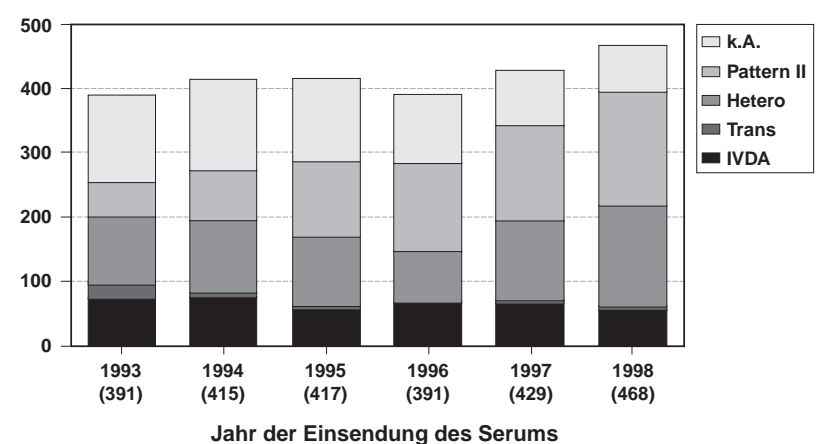

Abb. $3 \Delta$ Infektionsrisiken bei weiblichen HIV-Infizierten in Deutschland im Zeitverlauf seit 1993. Aufgeführt sind nur die von den meldenden Labors explizit als Erstdiagnosen bezeichneten Befunde k.A. =keine Angaben, Pattern II=Herkunft aus Hochprävalenzregion, Trans=Transfusionsempfänger, IVDA=intravenös Drogenabhängige

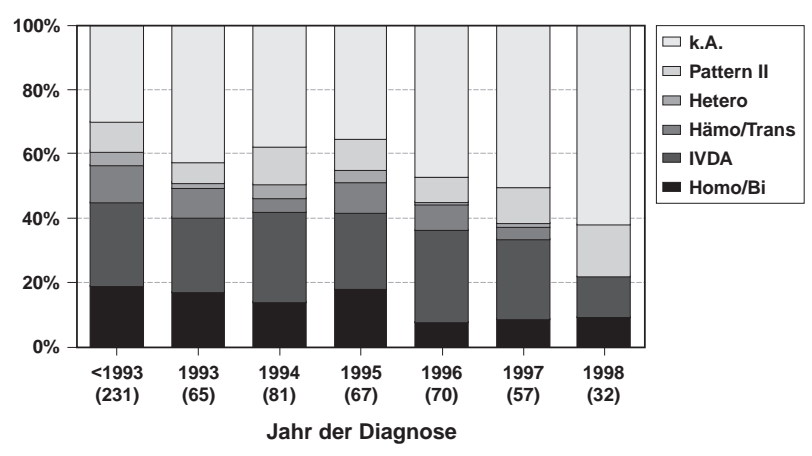

Abb. $2 \Delta$ Risiko des männlichen Partners bei weiblichen AIDS-Patienten, die sich über heterosexuelle Kontakte infiziert haben, im Zeitverlauf

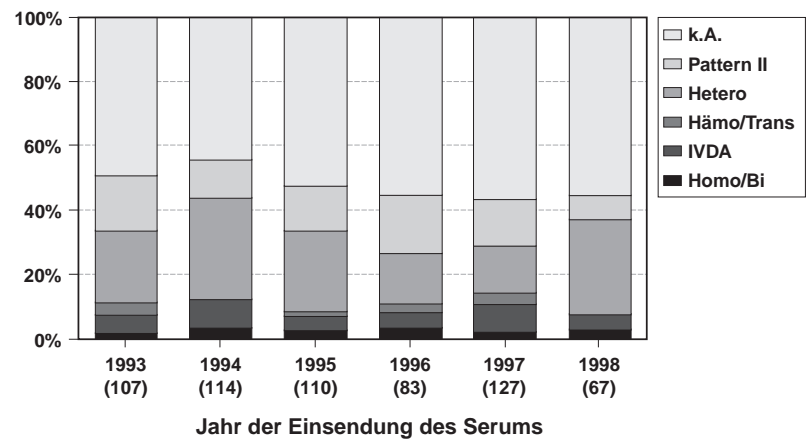

Abb. $4 \Delta$ Risiko des männlichen Partners von Frauen, die sich auf heterosexuellem Wege infiziert haben. Aufgeführt sind HIV-Erstdiagnosen seit 1993
Bei einer steigenden Zahl HIV-infizierter junger Frauen im gebärfähigen Alter müßte man eigentlich auch mit einer zunehmenden Zahl von Mutter-KindÜbertragungen der HIV-Infektion rechnen. Entgegen dieser Erwartung geht die Zahl der HIV-Infektionen bei Kindern zurück. Generell besitzt die Mutter-KindÜbertragung in den westlichen Industrienationen einen viel geringeren Stellenwert als in Entwicklungsländern zum einen wegen des geringeren Frauenanteils an den Infizierten, zum anderen wegen der niedrigeren Reproduktionsrate, die bei den zuerst betroffenen Gruppen von Frauen, i.v. Drogenabhängigen und Transfusionsempfängerinnen, nochmals niedriger liegt als in der weiblichen Durchschnittsbevölkerung.

Das in der zweiten Hälfte der achtziger Jahre häufig aufgrund mangelnder
Daten noch überschätzte Mutter-KindÜbertragungsrisiko und die fehlenden Therapiemöglichkeiten bewegten anfangs die Mehrzahl der HIV-infizierten Frauen, bei denen eine HIV-Infektion bereits vor einer Schwangerschaft bekannt war oder während der Schwangerschaft entdeckt wurde, zum Abbruch der Schwangerschaft. Die Skrupel der Schwangeren wurden durch sozialen Druck der Umgebung und oftmals auch der betreuenden Frauenärzte in Richtung auf eine Entscheidung zum Schwangerschaftsabbruch zusätzlich noch verstärkt. Spätestens ab Anfang 1994 änderte sich die Situation durch die Erkenntnis, daß eine antiretrovirale Therapie mit Zidovudin während der Schwangerschaft, unter der Geburt und in den ersten Lebenswochen das Übertragungsrisiko deutlich senken konnte.

\section{„Mit den verfügbaren Maßnahmen zur Prophylaxe der Mutter-Kind-Übertragung könnten HIV-Infektionen bei Kindern fast vollständig vermieden werden."}

Zeitgleich ergaben retrospektive Analysen der Mutter-Kind-Übertragungsraten in Deutschland, daß die frühzeitige Kaiserschnittentbindung am wehenfreien Uterus ebenfalls eine deutliche Senkung der Mutter-Kind-Übertragungsrate bewirkte. Schon lange bevor sich diese Erkenntnis in den internationalen Diskussionen durchsetzen konnte, etablierte sich in Deutschland die Kombination von Zidovudin-Prophylaxe und frühzeitiger Sektio als Behandlungsund Entbindungsstandard, mit dem die Mutter-Kind-Übertragungsrate auf unter $2 \%$ gesenkt wurde [2] (siehe auch 


\section{Leitthema:Mutter-Kind-Übertragung von Infektionskrankheiten}

\begin{tabular}{|c|c|c|}
\hline \multirow[t]{2}{*}{ AUT-Untersuchungszeitraum } & $\begin{array}{l}\text { Berlin } \\
1.01 .93-31.12 .97\end{array}$ & Niedersachsen \\
\hline & $81 / 141.149(0,57 / 1.000)$ & $51 / 375.714(0,14 / 1.000)$ \\
\hline & & Nur Hannover \\
\hline HIV-Status bei Geburt bekannt & $66 / 81(81 \%)$ & $15 / 23(65 \%)$ \\
\hline Bekannt vor Schwangerschaft & $35 / 66$ & $3 / 15$ \\
\hline Entdeckt während Schwangerschaft & $29 / 66$ & $12 / 15$ \\
\hline Serokonversion während Schwangerschaft & 3 & \\
\hline Entdeckt nach Entbindung & & $2 / 23$ \\
\hline Diagnosedatum unbekannt & 2 & 2 \\
\hline Infektionsrisiken & & \\
\hline IVDA & 35 & 4 \\
\hline Hetero & 15 & 3 \\
\hline Herkunft aus Hochprävalenzgebiet & 6 & 12 \\
\hline Bluttransfusion & 5 & 0 \\
\hline unbekannt & 5 & 0 \\
\hline
\end{tabular}

AUT-Positivrate in Bayern 1995/96:34/182.808 (0,19/1.000)

Davon München: 19/33.512 (0,57/1.000)

Beitrag von A. Schäfer et al. in diesem Heft).

Mit diesem Vorgehen könnte theoretisch die Mutter-Kind-Übertragung in Deutschland nahezu eliminiert werden, vorausgesetzt, Schwangerschaften HIVinfizierter Frauen werden rechtzeitig erkannt. In den deutschen Mutterschaftsrichtlinien wird die Durchführung eines HIV-Antikörpertestes in der Schwangerschaft empfohlen. Diese Empfehlung wird jedoch nicht hundertprozentig be- folgt, und oftmals findet die ebenfalls empfohlene Beratung und Aufklärung der Schwangeren nicht statt, so daß ein Teil der Untersuchungen ohne explizite Ankündigung und Kenntnis der Schwangeren durchgeführt wird.

Die in zwei Bundesländern, Berlin und Niedersachsen, durchgeführten AUT-Studien (AUT=anonyme, unverknüpfbare Testung) bei denen Proben, die zum Neugeborenenscreening auf Stoffwechselkrankheiten gewonnen wer- den, anonymisiert und auf HIV-Antikörper untersucht werden, erlauben einen Abgleich zwischen den bei den Behandlungszentren bekannten HIV-Schwangerschaften und der Gesamtzahl der HIV-Schwangerschaften. Dieser Abgleich zeigt, daß in Berlin ca. $80 \%$, in der niedersächsischen Landeshauptstadt Hannover ca. $65 \%$, der HIV-Schwangerschaften bekannt sind (Tabelle 1). Hochgerechnet auf ganz Deutschland kann man damit rechnen, daß in ungefähr $70 \%$ der Schwanger-

Tabelle 2

Abschätzung der in Deutschland jährlich geborenen HIV-infizierten Kinder

Geschätzte Zahl ausgetragener Schwangerschaften HIV-positiver Mütter/Jahr

\section{Vergleichswerte in anderen Ländern}

HIV-Status der Mutter bei Entbindung bekannt

Großbritannien 1995/96: 
Tabelle 3

Meldungen gemäß Laborberichtsverordnung* mit Angabe des Übertragunsgrisikos Mutter-Kind

\begin{tabular}{lccccc} 
& 1994 & 1995 & 1996 & 1997 & 1998 \\
\hline $\begin{array}{l}\text { Alle Meldungen unter Ausschluß } \\
\text { erkennbarer Doppelmeldungen }\end{array}$ & 97 & 69 & 63 & 50 & 52 \\
Definitive Erstmeldung & 74 & 52 & 52 & 33 & 25 \\
0-11 Monate & 68 & 43 & 36 & 26 & 3 \\
1-4 Jahre & 3 & 9 & 8 & 1 & 1 \\
5-9 Jahre & 3 & - & 3 & - & - \\
10-12 Jahre & - & - & 4 & & \\
\hline
\end{tabular}

* Grundsätzliche Problematik bei den Labormeldungen ist der nicht vollständig erkennbare Anteil von Doppelmeldungen. Bei den Meldungen über Kinder im Alter von 0-ca. 18 Monaten kommt als zusätzliches Problem hinzu, daß der Antikörpernachweis gemeldet wird, nicht die Infektion!

schaften HIV-infizierter Frauen die HIVInfektion der Schwangeren entweder bereits vor der Schwangerschaft bekannt war oder bei entsprechenden Untersuchungen in der Schwangerschaft entdeckt wird. Falls diese Annahme zutrifft, müßte die Zahl der jährlich mit HIV-Infektionen geborenen Kinder unter zehn liegen (Tabelle 2).

Die Datenquelle, die man zur Validierung dieser Abschätzung heranziehen sollte, die Labormeldepflicht, versagt bisher in diesem Zusammenhang, weil sie lediglich die Meldung positiver Antikörperbefunde vorsieht, die jedoch beim Neugeborenen einer HIV-infizierten Mutter bis zum Alter von 15 bis 18 Monaten keine Aussage zum Infektionsstatus des Kindes zulassen. Es bleibt daher weitgehend unklar, was sich hinter den Meldungen positiver Antikörperbefunde von Neugeborenen im ersten Lebensjahr verbirgt. Erstmeldungen von Kindern in höheren Altersgruppen spiegeln bei perinatalem Infektionsrisiko hingegen mit großer Wahrscheinlichkeit diejenigen Kinder wider, bei denen die HIV-Infektion der Mutter erst nach der Entbindung bekannt wurde und/oder die klinisch auffällig werden. Die Zahl der Erstmeldungen perinatal HIV-infizierter Kinder im Alter zwischen einem und neun Jahren sank von elf im Jahre 1996 auf vier im Jahre 1998 (Tabelle 3).

\section{Fazit für die Praxis}

Trotz steigendem Anteil von Frauen an den HIV-Infizierten kann die MutterKind-Übertragung von HIV-positiven Frauen mit Kinderwunsch durch eine Kombination von antiretroviraler Prophylaxe und primärer Kaiserschnittentbindung auf unter $2 \%$ reduziert werden. Beide Maßnahmen können nur greifen, wenn die HIV-Infektion der Schwangeren bekannt ist. Spätestens im Rahmen der Schwangerschaftsvorsorgeuntersuchungen sollte daher jeder Schwangeren angeboten werden, einen HIV-Test durchzuführen. Dieses Angebot muß von einer qualifizierten Aufklärung und Beratung begleitet sein.

\section{Literatur}

1. AIDS/HIV Quartals- und Jahresberichte des Robert Koch-Instituts

2. Grosch-Wörner I, Arasteh K, Brockmeyer N, Friese K et al. (1998) Deutsch-Österreichische Empfehlungen zur HIV-Therapie in der Schwangerschaft (Stand Mai/Juni 1998).URL:http://www.rki.de/INFEKT/AIDS_STD/AZ.HTM

3. Nicoll A, McGarrigle C, Brady AR et al. (1998) Epidemiology and detection of HIV-1 among pregnant women in the United Kingdom: results from national surveillance 1988-96. BMJ 316:253-258

4. CDC (1998) Success in implementing PHS guidelines to reduce perinatal transmission of HIV - 1993, 1995, and 1996. MMWR 47:688-691 